\section{GRP-152 PROSPECTIVE STUDY ON THE USE OF RESTRICTED-USE ANTIBIOTICS: ERTAPENEM, LINEZOLID, TIGECYCLINE AND DAPTOMYCIN}

doi:10.1136/ejhpharm-2013-000276.152

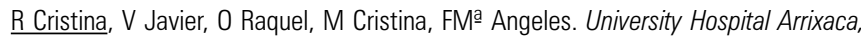
Pharmacist, Murcia, Spain

Background Because of antibiotic resistance problems and frequent inappropriate use of antimicrobial agents in hospitals, these drugs have often been the target of attempts to restrict their use.

Purpose To analyse the appropriateness of use of restricted-use antibiotics approved by the Drug and Therapeutics Committee (DTC).

Materials and Methods The study was conducted prospectively from October 2011 to February 2012 in our hospital.

We included all patients treated with restricted antibiotics. The data required for the clinical monitoring of patients were collected from the clinical history. The variables were: age of the patient and laboratory data, clinical service, justification of the indication and duration of treatment.

Results We included a total of 100 patients, of whom 39 were treated with ertapenem (39\%), 7 with tigecycline (7\%), 49 with linezolid (49\%) and 5 with daptomycin (5\%). The percentage of non-compliance, based on criteria for use approved by the DTC, was $14 \%$.

In analysing the results for Services we found that $90 \%$ of prescriptions that conformed to the approved DTC indications were prescribed by General Surgery, $81.8 \%$ by Internal Medicine, $55 \%$ by Infectious Diseases, $85 \%$ by Traumatology, $71.4 \%$ by Cardiovascular Surgery, $66 \%$ by Pulmonology, $50 \%$ by Urology and $100 \%$ by other services. The suitability was $92.3 \%$ for ertapenem, $85.71 \%$ for tigecycline, $83.67 \%$ for linezolid and $60 \%$ for daptomycin. The average duration of treatment was 4.5 days for ertapenem, 12.5 days for linezolid, 11 days for tigecycline and 18 days for daptomycin.

\section{Conclusions}

1. The appropriateness of use of restricted antibiotics as approved by the DTC, although acceptable, could be improved.

2. An antibiotic control programme between the Pharmacy and Infectious Diseases could improve the quality of patient care.

No conflict of interest.

\section{GRP-153 RECOMMENDATION FOR ERROR PREVENTION IN ANTICANCER DRUG TREATMENT}

doi:10.1136/ejhpharm-2013-000276.153

'S Ciampalini, 'L Guidotti, ${ }^{2} \mathrm{~L}$ Fabrizio, ${ }^{3} \mathrm{~A}$ Mandelli, ${ }^{1} \mathrm{~A}$ Ghirardini, ${ }^{1} \mathrm{~F}$ Bevere. 'Ministry of Health, Quality, Rome, Italy; ${ }^{2}$ SIFO, Pharmaceutical, Rome, Italy; ${ }^{3} \mathrm{FOFI}$, Pharmaceutical, Rome, Italy

Background Due to the high toxicity of anticancer drugs and their low therapeutic index, any errors in anticancer drug treatment can cause serious injury, even at approved doses. For this reason it is necessary have some national guidance to prevent errors during anticancer treatment.

Purpose To prevent errors in anticancer drug treatment, the Ministry of Health has developed a specific Recommendation addressed to healthcare workers (physicians, pharmacists and nurses).

Materials and Methods A working group consisting of pharmacists, oncologists, nurses, psychologists and patients representatives was set up. The same format of Recommendations made by $\mathrm{MoH}$ for Patient Safety was followed.

Results The Recommendation is addressed to Regions, Health facilities, clinical risk managers, healthcare workers; it finds application inside the Medical Oncology and Haematology Units of private and public hospitals, inside hospital pharmacies and at the patient's home. The Recommendation is to protect patients needing anticancer drug treatments. Highlight: the hospital should provide working instruments and develop a procedure including all instructions for proper anticancer drug management. They also have to: draw up a Patient Safety Training Plan, promote communication and integration among health professionals, provide accurate and complete information to patients and their families, consider the psychologi$\mathrm{cal}$ and emotional state of patients and give detailed indications for the delivery of drugs at discharge.

Conclusions The Recommendation is a reference for health professionals involved in handling anticancer drugs, providing information about the patient's objectives and expected benefits from treatment. The document provides guidance aimed at preventing errors that can occur during anticancer drug treatment and includes recommendations encouraging the promotion of clinical governance. A verification of this Recommendation is expected soon. Many experts gave their suggestions to facilitate its implementation.

No conflict of interest.

\section{GRP-154 RECONCILIATION AND DRUG INFORMATION TO GERIATRIC POLYMEDICATED PATIENTS AT DISCHARGE USING INFORMATION TECHNOLOGY}

doi:10.1136/ejhpharm-2013-000276.154

M Moro Agud, T Pérez Robles, M Ruano Encinar, R Menéndez Colino, MC Mauleón Ladrero, A Herrero Ambrosio. Hospital Universitario La Paz, Pharmacy, Madrid, Spain

Background Aging of the Spanish population increases the elderly patient consensus and demand for health care in hospitals. Elderly patients have particular characteristics that increase the risk of medication errors.

Purpose To establish a programme that involved medicines reconciliation and adapted drug information for elderly patients with polypharmacy at discharge. Several electronic resources were used in order to promote continuity of care and adherence to pharmacotherapy.

Materials and Methods Geriatricians selected patients according to three criteria: more than 70 years old, at least five medicines at discharge and some difficulty understanding them. Doctors electronically requested the Pharmacy Department to complete the developed 'Medication Information Form at Discharge.' A pharmacist reviewed the treatment prescribed at discharge and reconciled it with the patient's medicines during hospitalisation via electronic records. The pharmacist resolved discrepancies found with the physician. A visually appealing and understandable form was submitted electronically to be given to the patient.

Results From April 2011 to March 2012, this service was performed for 57 patients. Most of them were women (77.2\%) with a mean age of 88.5 (SD 6.2) years old. 555 Drugs were reported (9.7 drugs/patient) and 696 were reconciled (12.2 drugs/patient). 143 discrepancies were found (2.5 discrepancies/patient): 135 of them were justified (94.4\%) and the other 8 were medicines errors (0.014\%).

Conclusions Information technology enables pharmacists to undertake this work: improving communication between professionals, inserting the 'Medication Information Form at Discharge' into clinical documentation, enabling medicines reconciliation and adapting the information sheet to the geriatric population. This practise provides reconciliation of medicines that have been prescribed before, during and after hospitalisation. In summary, it is necessary to achieve adequate therapeutic adherence and to avoid administration errors, which may have consequences on patient health and increased costs

No conflict of interest. 